\title{
Spatio-Temporal Clustering of Firing Rates for Neural State Estimation
}

\author{
Austin J. Brockmeier, Student Member, IEEE, Il Park, Babak Mahmoudi, Student Member, IEEE, \\ Justin C. Sanchez, Member, IEEE and José C. Príncipe, Fellow, IEEE
}

\begin{abstract}
Characterizing the dynamics of neural data by a discrete state variable is desirable in experimental analysis and brain-machine interfaces. Previous successes have used dynamical modeling including Hidden Markov Models, but the methods do not always produce meaningful results without being carefully trained or initialized. We propose unsupervised clustering in the spatio-temporal space of neural data using time embedding and a corresponding distance measure. By defining performance measures, the method parameters are investigated for a set of neural and simulated data with promising results. Our investigations demonstrate a different view of how to extract information to maximize the utility of state estimation.
\end{abstract}

\section{INTRODUCTION}

In the analysis of neuronal recordings, there is a need to explain the data over time with a latent (state) variable. State estimation from multi-electrode neural data is a tool for characterizing the underlying network dynamics and can be used to find areas of interest (synchronization or correlation) over time. A common time course analysis tool for multi-trial recordings is the peri-event time histogram, but it averages over trials and treats each neuron separately whereas a state-estimation provides meaning to trial variability over an ensemble [1]. For applications such as brain-machine interfaces, it is applicable to find either discrete state estimation (e.g. when to start decoding for movement [2]) or continuous state estimation (state-based linear decoding [3]).

Work in state-estimation for multi-electrode recordings studies is based on dynamical models such as Hidden Markov Model [4], Kalman filter [3], or finite state machines [5]. The goals include an abstract dynamical model for visual pattern stimuli [1], finding synchronous activity beyond ensemble oscillations [6], and quasi-stationary states in network dynamics [7]. More recent work in brain-machine interfaces detect neural states to decode the neural data with respect to movement type [2] and plan timing [5]. A review of methods is in [8] and [9].

This work was supported in part by the Univ. Florida Graduate School Fellowship, the U.S. National Science Foundation Grant PFI-0650161, U.S Office of Naval Research Grant N00014-10-1-0375, and DARPA Grant N66001-10-C-2008.

A. Brockmeier and J. Príncipe are with the Department of Electrical and Computer Engineering, University of Florida, P.O. Box 116130 NEB 486, Bldg \#33, University of Florida, Gainesville, FL 32611 USA (e-mail: ajbrockmeier@ufl.edu, principe@cnel.ufl.edu)

I. Park and B. Mahmoudi are with the Department of Biomedical Engineering, University of Florida, Biomedical Sciences Building JG-56, P.O. Box 116131, Gainesville, FL 32611 USA (e-mail: memming@cnel.ufl.edu, babakm@ufl.edu)

J. Sanchez is with the Department of Pediatrics, Division of Neurology, University of Florida, P.O. Box 100296, JHMHC, Gainesville, FL 32610 USA (e-mail: jcs77@ufl.edu)
Besides dynamical models, static analysis such as principal-component analysis (PCA) and local-linear embedding (LLE) [10] find projections of neuronal trajectories which reduce the dimensionality of the recordings while maintaining a sense of trial trajectories [11]. However, PCA in particular and to some extent LLE fail to exploit the temporal structure of the data [8].

If the goal is to find a discrete set of states using unsupervised methods, then PCA and LLE fail to provide quantization in time and Hidden Markov models suffer from the need of prior training. Another method is to take static analysis one step further and apply clustering to neural trajectories. Besides clustering of dynamical models by [12], clustering to find temporal structure seems to be an unexplored approach to discrete-state estimation in neural data.

Our approach to neural state estimation is to apply unsupervised clustering. This approach is independent of training data and can be modified to an online method that builds the state-space iteratively. The method defines a joint domain of neuron firing rates over time for clustering. At each sample, a vector is built with all the sampled neurons estimated firing rates at a time step and some previous values. We investigate a novel divergence measure in the combined spatio-temporal rate space to cluster the data. We further define information theoretic measures of performance to rate the generated state estimation across method dependent parameters.

The method is demonstrated on a simple simulated data set and neural recordings from the Nucleus Accumbens region of a rat during a behavioral task. We show that the information provided by the states is consistent across trials and states represent known timings. The results suggest that by clustering in the spatio-temporal space discrete states are generated that correspond to meaningful dynamics in neural activities without training data.

\section{METHODS}

\section{A. Data Representation}

Time embedding is used to define a spatio-temporal space for the clustering. It is hypothesized that the neural data can be grouped into meaningful clusters defining the state of the ensemble. At each time step a vector is composed of the spike counts of all neurons over a window of time

$$
\begin{aligned}
x_{i}= & {\left[r_{1}(i), r_{2}(i), \ldots, r_{n}(i), r_{1}(i-1), r_{2}(i-1), \ldots,\right.} \\
& \left.r_{n}(i-1), r_{n}(i-2), \ldots, r_{n}(i-L+1)\right] \in \mathbb{Z}_{+}^{n \cdot L}
\end{aligned}
$$

where $r_{v}(i)$ is bin count for neuron $v$ at time step $i, n$ is the number of neurons, and $L-1$ is the maximum lag of the time 
embedding. We analyze a set $X_{T}=\left\{x_{1}, \ldots x_{T}\right\}$ of $T$ such vectors. The goal is to assign an estimated clusters/states defined by the spike count vectors at each time step.

In order to cluster, we need a distance measure which works across different neurons at different time steps. The simplest approach would be to treat all dimensions as equal with Euclidean distance. This treats the neurons and successive times equivalently. Another approach is to use smaller weights for greater time lags. The intuition behind this approach is that it will put emphasis on the recent data instead of treating all time lags in the window equally, while still providing more information than instantaneous firing rate. A weighted Euclidean distance is of the form

$$
d_{w}\left(x_{i}, x_{j}\right)=\left(\sum_{l=0}^{L-1} w_{l} \sum_{v=1}^{n}\left(r_{v}(i-l)-r_{v}(j-l)\right)^{2}\right)^{\frac{1}{2}} .
$$

The weights for successive lags are decayed exponentially $w_{l}=e^{\frac{-l}{L-1}}$. The time constant is set to the maximum lag $L-1$.

If we assume the underlying variability is an inhomogeneous Poisson process, a common model for binned spike counts, then the mean and variance of the binned spike counts are equal. In other words, for higher firing rates, the variance is higher. Therefore it is unfair to use a Euclidean distance between the count values. Instead, we propose to estimate the mean of the Poisson process by the count sample itself, and use Kullback-Leibler divergence between the corresponding Poisson processes. The KullbackLeibler divergence between two Poisson processes with rate $\lambda_{1}, \lambda_{2}$ is $D_{K L}\left(P_{\lambda_{1}} \| P_{\lambda_{2}}\right)=\lambda_{2}-\lambda_{1}+\lambda_{1} \log \frac{\lambda_{1}}{\lambda_{2}}$ [4]. To form a symmetric distance measure, we use $D\left(\lambda_{1}, \lambda_{2}\right)=$ $D_{K L}\left(P_{\lambda_{1}} \| P_{\lambda_{2}}\right)+D_{K L}\left(P_{\lambda_{2}} \| P_{\lambda_{1}}\right)=\left(\lambda_{1}-\lambda_{2}\right) \log \frac{\lambda_{1}}{\lambda_{2}}$. Again we apply exponentially decayed weights for the time lags to form a distance measure

$$
d_{w}\left(x_{i}, x_{j}\right)=\sum_{l=0}^{L-1} w_{l} \sum_{v=1}^{n} D\left(r_{v}(i-l), r_{v}(j-l)\right) .
$$

\section{B. Clustering}

Spectral clustering is chosen as the method of clustering because of proven successes in a variety of domains [13]. It is a graph theoretic clustering method using an affinity matrix as the weights of the edges which define the graph. The affinity matrix $A$ is constructed from applying a kernel function $K_{\tau}$ to the distances, $A_{i j}=K_{\tau}\left(d_{w}\left(x_{i}, x_{j}\right)\right)$. Spectral clustering tries to cut the graph defined by this matrix into $k$ subgraphs so each subgraph is maximally connected (minimum edge distances). This cut is suboptimal, but it is guaranteed to be an approximation of the optimal partition [13]. The formulation for the algorithm is in [14]. We use a Gaussian kernel $K_{\tau}(d)=1 /(\sqrt{2 \pi} \tau) \exp \left(d^{2} /\left(2 \tau^{2}\right)\right)$. As $\tau$ increases, distances between points relate to decreasing values in the affinity matrix, $d\left(x_{i}, x_{j}\right) \rightarrow \infty \Longrightarrow A_{i j} \rightarrow 0$. Ideally, points in the same cluster have affinity entry of 1 and 0 otherwise. We can automatically find the value of $\tau$ by using the clustering with the 'tightest' clusters [14]. A measure for tightness is defined in (3) which goes to zero for clusters with zero variance. The method of clustering is computationally straightforward, but still requires a userdefined number of clusters.

To speed computation and demonstrate robustness we cluster only a fraction $t$ out of a possible $T$ samples. The $t$ samples are divided to $k$ clusters $S_{1}, S_{2}, \ldots, S_{k} \in S$. For each cluster $S_{j}$ we compute the mean $\bar{x}_{j} \in R^{N_{n} \cdot L}$ over all sample points assigned to that cluster. Then we assign all $X_{T}$ samples to the closest cluster defined by our distance measure. To summarize, the user-defined parameters are the time embedding depth $L$, cluster count $k$, the time constant for the weighting, and the distance measure (1) or (2).

\section{Performance Measures}

To evaluate the results of clustering and of state estimation we define a set of measures for tightness of the clustering (3), consistency of state estimates given known timing (4), and optimizing state estimates with respect to known information (5).

We measure the tightness of the clustering as the average variance of points within a cluster divided by the variance in the cluster centers.

$$
\frac{\bar{\sigma}_{x}^{2}}{\sigma_{S}^{2}}=\frac{\sum_{j=1}^{k} \frac{1}{\left|S_{j}\right|} \sum_{x_{i} \in S_{j}}\left(x_{i}-\bar{x}_{j}\right)^{2}}{\sum_{j=1}^{k}\left(\bar{x}_{j}-\bar{X}\right)^{2}}
$$

where $S_{j}$ is cluster with mean $\bar{x}_{j}$ and $\bar{X}$ is mean of the cluster means.

To measure the consistency of the states, we assume a discrete random variable $Y=\left\{y_{1}, y_{2}, \ldots, y_{M}\right\}$ which influences the state. We measure if the states during these times are meaningful with respect to $Y$. The goal of the method is to obtain states that represent as much information about $Y$ as possible. If $Y$ is known at some times across trials to be $y$, then at these times the set of state estimates should identify $y$. A measure of this is the conditional entropy $H(S \mid y)$. It will be lowest when all samples share the same states each time $Y=y$ and will be maximized when there is a uniform distribution of states given $Y=y$. The conditional entropy of the state estimates when $Y=y$ is defined as

$$
H(S \mid y)=-\sum_{j=1}^{k} P\left(S_{j} \mid y\right) \log \left(P\left(S_{j} \mid y\right)\right)
$$

where $P(S \mid y)$ is the conditional probability mass function of the states estimated by the relative state frequency given the set of samples $X_{y}$ where $Y=y \cdot P(S \mid y)$ is estimated as $\hat{P}\left(S_{j} \mid y\right)=\frac{1}{\left|X_{y}\right|}\left|\left\{i: x_{i} \in X_{y} \cap S_{j}\right\}\right|$.

As a global criterion, we want to minimize the unconditional entropy in the state estimates $H(S)$ while maximizing the relevant information in $S$ relative to $Y$. This can be framed similar to the information bottleneck method [15]; we want to minimize the entropy of our states $H(S)$ while maximizing the mutual information between the state and the information signal $Y$,

$$
I(S ; Y)=\sum_{y \in Y} \sum_{S_{j} \in S} P\left(S_{j}, y\right) \log \left(\frac{P\left(S_{j}, y\right)}{P\left(S_{j}\right) P(y)}\right)
$$


where $P\left(S_{j}, y\right)=\hat{P}(Y=y) \hat{P}\left(S_{j} \mid X_{y}\right)=\frac{\left|X_{y}\right|}{\left|X_{T}\right|} \hat{P}\left(S_{j} \mid X_{y}\right)$

In the end, we have a trade off between minimizing $H(S)$ and maximizing $I(S ; Y)$; forming a criterion with weight $\beta$ for these quantities we have

$$
J(S, Y)=H(S)-\beta I(S ; Y) .
$$

This simply introduces a new variable $\beta$, and there is an optimum for any value [15]. However, $\beta=1 \Longrightarrow$ $J(S, Y)=H(S \mid Y)$ which has the information theoretic idea of minimizing the entropy in $S$ when $Y$ is known. If we went to the work of clustering we want at least an order of magnitude more information about $Y$ than the information it takes to encode $S$, so we set $\beta$ to be 100. In summary, given data we want to minimize (3) in the clustering and then given an information signal $Y$ which is discrete and can partition time, we want to find a state estimation $S$ such that $J(S, Y)$ is as small as possible.

\section{RESULTS}

\section{A. Simulated Data}

To test the method and measures we construct a simulated dataset consisting of an inhomogeneous Poisson process, a common model for binned spike counts. The process mean is controlled by a state variable taking five states. For three states the mean is constant $(5,10$, or 20$)$ and two states the mean increases (or decreases) linearly between 5 and 10 . The duration of each state is 20 time steps and transition between states is random with the restriction that the mean rate cannot instantaneously change. Since we know the true sequence of states we can evaluate the performance over different method parameters $k, L$, and distance measure. In addition, our method can produce an estimated output at time $i$ given the state at $i$ and its respective mean $\bar{x}$ by $\sum_{l=1}^{L} w(l) \bar{x}(l)$. The simulated data and an example result is shown in Fig. 1 and performance over various parameters is shown in Fig. 2. From the figure it is clear that given our performance criterion and the desired cardinality of the $S$ an optimum time lag can be found.

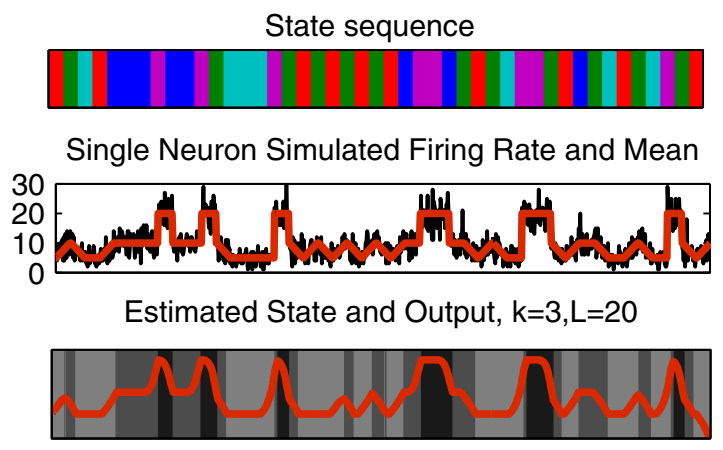

Fig. 1. A random state sequence was created (top) which controls the mean of a Poisson process. The mean moves between 5,10 , or 20 or stays the same at each state as seen by the plot of mean and a realization (middle). An estimate of state and output is shown in (bottom). Having a time history encoded in each cluster mean, $\bar{x}$, allows an accurate estimation with only three states.

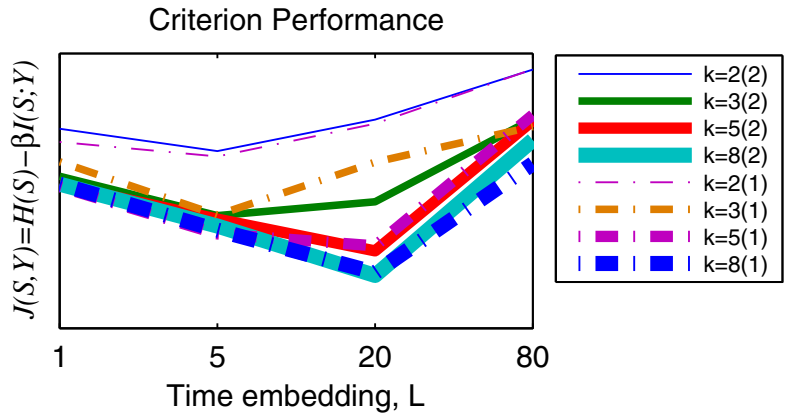

Fig. 2. The performance criterion for different time embeddings $L$, number of states $k$, and distance measure (2) or (1). Because of the large value of $\beta=100$ increasing number of states is penalized less than providing information about the true state. However, there is an optimum time embedding of 5 for 2 or 3 states and 20 for 5 or 8 . At 20, a state is able to capture the time course for a full state cycle.

\section{B. Nucleus Accumbens Data}

We apply the clustering method to microwire neuronal data recorded chronically from the Nucleus Accumbens of a rat. Before implantation, the rat was trained in a two-lever choice task. By pressing the target lever, cued by LEDs, the rat received $0.04 \mathrm{~mL}$ of water. Each trial was initiated by the rat with a nose poke. There are separate trials for pressing left and right levers and the time of cue and reward is known. The data used in the analysis was from a single day's recording. During the recording there were 83 trials; on 33 trials the rat was required to press the left lever and on 50 trials the rat was required to press the right lever to receive the reward. After spike sorting, there were 41 neurons isolated. In the analysis we used a bin size of $100 \mathrm{~ms}$ with 120 bins per trial (12 s per trial). Two trials from each side were used in clustering (the choice had little impact on results as trial timings were consistent); the remaining samples are assigned to the state corresponding to the nearest cluster center.

The data from Nucleus Accumbens has the temporal structure as is seen in a single trial for the right lever Fig. 3. An example state estimation finds consistently across trials

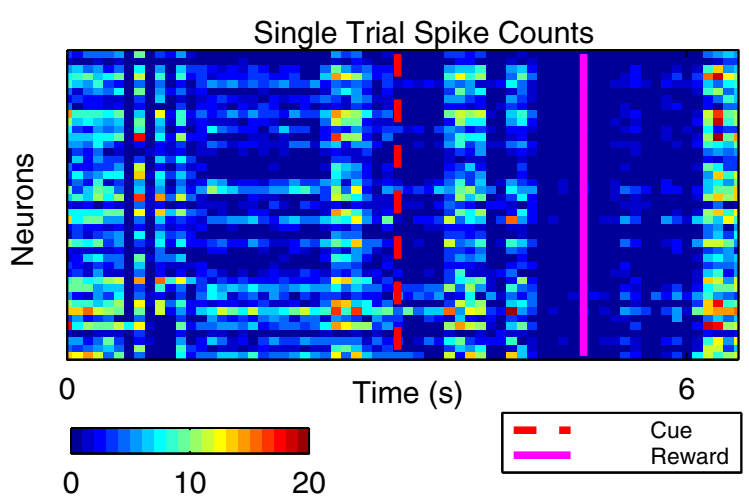

Fig. 3. The firing rate (bin counts with $100 \mathrm{~ms}$ bins) for a single trial of right lever task. There is temporal structure with respect to the task timings, but there is a diversity across the neurons. 


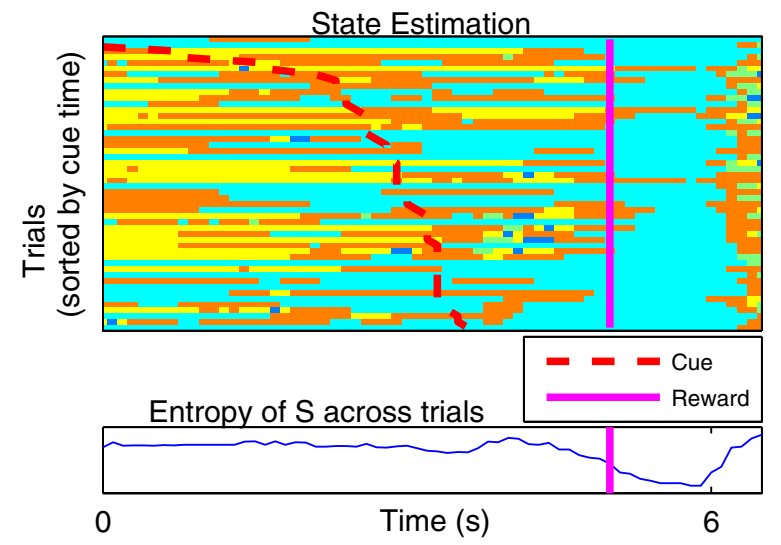

Fig. 4. The state estimates for each time step across right lever trials (top). The color corresponds to an arbitrary state label. The clustering was performed with $k=6$ clusters, time window of $L=20$, and divergence measure (2). The entropy of the set of states at each time point $H\left(S^{t}\right)$ goes down approaching the reward time (bottom). Besides consistency at each point approaching and after the reward, there is a pattern in the transitions (yellow,orange,cyan).

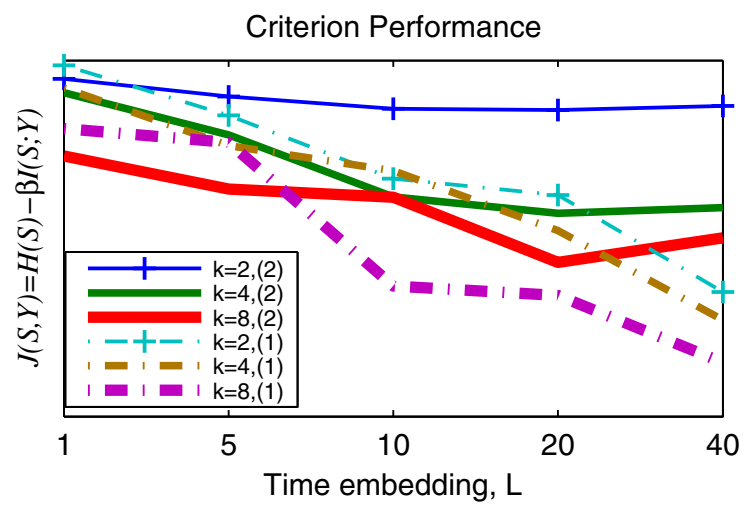

Fig. 5. The performance criterion for different time embeddings $L$, number of states $k$, and distance measure (2) or (1). Note that with (2) there is local minimum at a time embedding of 20 , while with (1) a longer window (greater than cue-reward interval) is still better.

Fig. 4. As shown in the figure the entropy of the states $H(S)$ at a given time across the trials decreases approaching the reward. Using the known timings we define $Y$ to be $y_{1}$ outside of cue and reward timing, $y_{2}$ during left trial, and $y_{3}$ during right trials and calculate (6) to compare across the user defined parameters of time embedding $L$, number of states $k$, and distance measures (1) or (2). The results are shown in Fig. 5. As in the simulated data, the large $\beta$ causes the criterion to improve for increasing the number of states. However, for the divergence measure there is minima with respect to $L$ the number of previous bins for each sample.

\section{CONCLUSION}

The results presented demonstrate a new method for state estimation from unsupervised clustering of neural data. The method is able to successfully capture trends in the data corresponding to known timings. With the simulated data, the algorithm is able to produce a generative output and sequence of state estimates similar to the true values. In addition, it is able to capture trends in data with a small number of states, something a HMM cannot do without breaking the Markov assumption, and it does this with no supervised initialization or data segmentation. The information theoretic measures we used can rate performance across method parameters, but the method needs refinement to avoid userdefined parameter choices of number of clusters and time embedding. A possible approach is to automatically tune the parameters in the distance measure to optimize the state information. Another consideration is applying a weighting to the neurons as currently they are taken to be independent and equally important.

One use of this method for state estimation is to automatically segment the data before applying other processing to the data such as non-stationary modeling or regression. The next step is transforming the state characterization and estimation from offline clustering to an online method.

\section{REFERENCES}

[1] G. Radons, J. Becker, B. Dülfer, and J. Krüger, "Analysis, classification, and coding of multielectrode spike trains with hidden Markov models," Biol. Cybern., vol. 71, no. 4, pp. 359-373, 1994.

[2] K. Shenoy, D. Meeker, S. Cao, S. Kureshi, B. Pesaran, C. Buneo, A. Batista, P. Mitra, J. Burdick, and R. Andersen, "Neural prosthetic control signals from plan activity," Neuroreport, vol. 14, no. 4, p. 591 , 2003.

[3] W. Wu, M. Black, D. Mumford, Y. Gao, E. Bienenstock, and J. Donoghue, "Modeling and decoding motor cortical activity using a switching Kalman filter," IEEE Trans. Biomed. Eng., vol. 51, no. 6, pp. 933-942, 2004.

[4] I. Gat, N. Tishby, and M. Abeles, "Hidden Markov modelling of simultaneously recorded cells in the associative cortex of behaving monkeys," Netw. Comput. Neural Syst., vol. 8, no. 3, pp. 297-322, 1997.

[5] N. Achtman, A. Afshar, G. Santhanam, B. Yu, S. Ryu, and K. Shenoy, "Free-paced high-performance brain-computer interfaces," J. Neural Eng., vol. 4, no. 3, p. 336, 2007.

[6] J. Deppisch, K. Pawelzik, and T. Geisel, "Uncovering the synchronization dynamics from correlated neuronal activity quantifies assembly formation," Biol. Cybern., vol. 71, no. 5, pp. 387-399, 1994.

[7] M. Abeles, H. Bergman, I. Gat, I. Meilijson, E. Seidemann, N. Tishby, and E. Vaadia, "Cortical activity flips among quasi-stationary states," Proc. Natl. Acad. Sci. U. S. A., vol. 92, no. 19, p. 8616, 1995.

[8] M. Churchland, B. Yu, M. Sahani, and K. Shenoy, "Techniques for extracting single-trial activity patterns from large-scale neural recordings," Curr. Opin. Neurobiol., vol. 17, no. 5, pp. 609-618, 2007.

[9] L. Paninski, Y. Ahmadian, D. Ferreira, S. Koyama, K. Rahnama Rad, M. Vidne, J. Vogelstein, and W. Wu, "A new look at state-space models for neural data," J. Comput. Neurosci., pp. 1-20, 2009.

[10] S. Roweis and L. Saul, "Nonlinear dimensionality reduction by locally linear embedding," Science, vol. 290, no. 5500, p. 2323, 2000.

[11] B. Yu, A. Afshar, G. Santhanam, S. Ryu, K. Shenoy, and M. Sahani, "Extracting dynamical structure embedded in neural activity," Adv. Neural Inf. Process Syst., vol. 18, p. 1545, 2006.

[12] S. Darmanjian and J. Principe, "Boosted and linked mixtures of HMMs for brain-machine interfaces," EURASIP J. Adv. Signal Proces., vol. 2008, no. 19, 2008.

[13] D. Spielman and S. Teng, "Spectral partitioning works: Planar graphs and finite element meshes," Linear Algebra Appl., vol. 421, no. 2-3, pp. 284-305, 2007.

[14] A. Ng, M. Jordan, and Y. Weiss, "On spectral clustering: Analysis and an algorithm," Adv. Neural Inf. Process Syst., vol. 2, pp. 849-856, 2002.

[15] N. Tishby, F. Pereira, and W. Bialek, "The information bottleneck method," Proc. 37th Allerton Conf. Communication, Control and Computing, pp. 368-377, 1999. 